International Journal of Case Reports
(ISSN:2572-8776)

\title{
Miller Fisher syndrome associated with respiratory failure
}

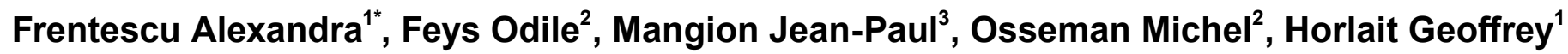 \\ ${ }^{1}$ Intensive Care Unit, Catholic University of Louvain, Belgium. \\ ${ }^{2}$ Neurology Deparment, Catholic University of Louvain, CHU UCL Namur, Avenue du Docteur G. \\ Therasse 1, 5530 Yvoir, Belgium. \\ ${ }^{3}$ Emergency Department, Catholic University of Louvain, CHU UCL Namur, Avenue du Docteur G. \\ Therasse 1, 5530 Yvoir, Belgium.
}

\begin{abstract}
Miller Fischer syndrome (MFS) is a rare clinical variant of GuillainBarre syndrome. The diagnosis suspected primarily on clinical ${ }^{*}$ Correspondence to Author: trial of areflexia, ophtalmoplegia and ataxia, is confirmed by the Frentescu Alexandra mandatory presence of antiganglioside antibodies (anti GQ1b). An acute onset is typical of MFS, beginning with neurological symptoms following a respiratory or digestive infectious illness. The therapeutic options are either the plasmapheresis or the Intensive Care Unit, Catholic University of Louvain, CHU UCL Namur, administration of intravenous immunoglobulin (IVIG). Although Avenue du Docteur G. Therasse 1, 5530 Yvoir, Belgium.

rare, in certain cases the patients present with respiratory symptoms needing intensive care. We report the case report How to cite this article: of a patient which presented with the classical triad of MFS but Frentescu Alexandra, Feys Odile, also with rapid progressive respiratory failure due to bilateral Mangion Jean-Paul, Osseman Micvocal cords palsy and general muscle weakness. He needed hel, Horlait Geoffrey. Miller Fisher srespiratory mechanical support. The patient received a treatment yndrome associated with respiratory by IVIG without any improvement in symptomatology. Eventually failure. International Journal of Cashe completely recovered and he was discharged from the e Reports, 2020; 4:173. hospital 3 month later.
\end{abstract}

Keywords: Miller Fisher syndrome; Atypical; Dysphonia; Respiratory failure

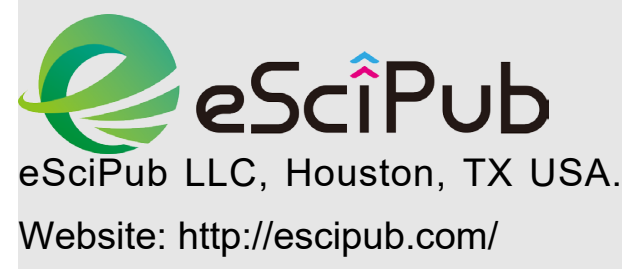




\section{Introduction}

Miller Fisher syndrome (MFS) is a rare variant of Guillain-Barre syndrome (GBS) which occurs in 1 to $5 \%$ of all GBS cases in Western countries with the notable exception of Japan, where it makes up to $25 \%$ of all GBS cases. It is twice as common in men as in women and commonly presents itself with areflexia, ophthalmoplegia and ataxia. ${ }^{[1]}$ Rarer symptoms include: dysesthesia; blepharoptosis; facial and bulbar palsies and pupillary abnormalities; mild motor weakness and micturition disturbances. ${ }^{[4,5]}$ Unlike the classic ascending weakness or paralysis typically seen in GBS, the neurological deficit follows a descending pattern starting with the head and moving downwards. ${ }^{[5]}$ An acute onset is usual, with neurological symptoms beginning approximately 8-10 days (range 1-30) following a respiratory or gastrointestinal $(\mathrm{Gl})$ infection with Haemophilus influenzae and Campylobacter jejuni being the most frequently identified culprits. Other associated pathogens include: $\mathrm{Myco}$ plasma pneumoniae, Cytomegalovirus, Epstein Barr Virus and the Human Immunodeficiency Virus. ${ }^{[3]}$ Upper respiratory symptoms precede the neurological ones in $76 \%$ of MFS cases with only $4 \%$ of patients having had preceding Gl symptoms. ${ }^{[6]}$ The disease progresses until the clinical nadir is reached approximately 6 days (range 221) after the initial neurological symptoms. Recovery is marked by gradual improvement and often resolution of the symptoms although rarely, serious complications such as respiratory failure or cardiac arrhythmias have been reported. ${ }^{[7]}$ Ataxia and ophthalmoplegia resolve within 1-3 months after onset and near complete recovery is expected within 6 months. Areflexia may persist but is not associated with functional disability. [3]

The diagnosis of MFS is primarily a clinical one and is confirmed by the presence of the serum anti-GQ1b antibodies that are present in 85 to $90 \%$ of the cases. ${ }^{[2,8]}$ The ganglioside GQ1b can be found in abundance in the paranodal region of the extramedullary portion of the oculomotor, abducens and trochlear nerves which makes these nerves the ones mostly affected by the self- reactive anti-GQ1b antibodies. 'Molecular mimicry' is believed to be the main pathogenic mechanism underlying the presence of these antibodies. ${ }^{[6]} \mathrm{MFS}$ is a rule-out diagnosis with a number of other pathologies including: GBS, Bickerstaff 's brainstem encephalitis, brainstem stroke, myasthenia gravis, Wernicke's encephalopathy and thyroid eye disease needing to be excluded beforehand. ${ }^{[3,6]}$

The disease is self-limiting with plasmapheresis and intravenous immunoglobulin therapy (IVIG) being controversial treatment options for which evidence of effectiveness remains limited. ${ }^{[9]}$

\section{Case report}

A 60-year-old Caucasian man presented to the emergency department with an episode of vomiting, dysphonia and hypertension. He also complained of vertigo and of feeling like "walking on cotton-wool". A couple of weeks prior to presentation he had experienced a few episodes of diarrhoea. His past medical history included chronic hypertension, type II diabetes, obesity and Scheuermann's disease.

On physical examination his vital signs were all in the normal ranges with the exception of arterial hypertension. His Glasgow Coma Score (GCS) was 15 and the neurological examination revealed mild dysphonia without other clinical signs suggestive of a vocal-cord paralysis, incomplete oculomotricity without clear signs of ophthalmoplegia, bilateral hypopallesthesia at the level of the malleoli and only on the right at the metacarpophalangeal level with symmetrically diminished lower limb reflexes and diminished right upper limb reflexes. A brain computed tomography (CT) scan ruled out a cerebrovascular accident and all blood tests were normal. The patient was discharged home with a suspicion of MFS and outpatient neurology follow-up.

He presented again the following day at the emergency department with worsening symptomatology. He had almost complete ophthalmoplegia, symmetrical pupils with a sluggish reaction to light, dysphonia, apallesthesia and areflexia of all limbs together with severe ataxia. The patient was still oriented to time, place and 
person with a GCS of 15 . Because of the patient's lumbar spine pathology an attempted lumbar puncture was unsuccessful. The patient was subsequently admitted to the neurology ward with MFS being retained as the probable diagnosis. A few hours following admission his dysphonia worsened and he was transferred to our intensive care unit (ICU) for respiratory surveillance. A vocal-cord examination revealed bilateral vocal-cord paralysis which later on that day necessitated oro-tracheal intubation following worsening respiratory failure. No limb muscle deficit was present at the time of admission to our unit.

All laboratory tests including a complete blood count, a metabolic panel and cardiac markers were normal. Serum cyanocobalamin, folic acid levels and thyroid hormone levels were also normal. Serum Campylobacter jejuni IgG and Cytomegalovirus IgG were positive however a serum Polymerase Chain Reaction test (PCR) failed to reveal the presence of Cytomegalovirus. Results obtained later showed the presence of antiGQ1b antibodies. Electromyography (EMG) showed a demyelinating polyneuropathy compatible with a diagnosis of both MFS and GBS. The initial diagnosis of MFS was based on the clinical presentation and the EMG result and was days later confirmed with the specific serum antibodies. It was considered to be a severe form of MFS because of its progressive extension involving all motor and mixed cranial nerves and general muscle weakness. The patient received a cycle of IVIG during five days without any clear improvement in symptomatology. He progressively developed general muscle weakness and bilateral facial palsy and also showed signs of autonomic dysfunction with episodes of bradycardia and hypertension. The weaning from mechanical ventilatory support was difficult with the patient necessitating a tracheostomy. Vocalcord examination revealed bilateral cord palsy with the cords in an adducted position.

Because of the lack of any significant improvement, a second cycle of IVIG was started on the third week following presentation. The treatment was interrupted on the third day because of severe clinical deterioration with worsening muscle weakness and the patient not being able to move any of his limbs, jaw or tongue. His pupils reacted more sluggishly to light and he had bilateral facial palsy with the EMG showing a deterioration of his polyneuropathy.

During the fourth week following admission, the neurological status of the patient started to progressively spontaneously improve and he was discharged from the ICU by the end of the same week. Thereafter, he spent one month in the neurology ward and another month in a neurorehabilitation unit. His vocal-cord paralysis completely recovered and he did not need the tracheostomy any longer.

\section{Discussion}

There are some aspects of the case we are presenting that are worth emphasising. Firstly, the main reason the patient presented to emergency room was because of dysphonia and a single episode of vomiting meaning that atypical presentations of MFS are possible. Although there was no recurrence of vomiting, the dysphonia eventually worsened leading to respiratory failure and necessitating orotracheal intubation.

Secondly almost all the motor and mixed cranial nerves were affected. In addition to the ophthalmoplegia typical of MFS, our patient also had bilateral facial palsy, deglutition problems due to throat and tongue muscle weakness and an inability to move the jaw.

Thirdly the patient developed flaccid quadriplegia with neurological symptoms progressing in a descending or top-down pattern, like an inversed GBS. This makes us wonder whether this was not a Miller-Fischer-Guillain-Barre overlap syndrome.

Fourthly the empirical treatment with IVIG did not seem to influence the evolution of the disease in the case we are reporting. When the treatment was started on admission the symptoms of the patient continued to worsen and during the second cycle of IVIG the symptomatology worsened so suddenly that a decision to stop the treatment was made.

\section{Conclusion}

This case highlights an atypical presentation of 
MFS. MFS is a rare variant of GBS. The mainly feature is the triad of ophthalmoplegia, areflexia and ataxia but atypical symptoms are also possible. Diagnosis is based mainly on clinical presentation and findings. The diagnosis is confirmed with the presence serum anti-GQ1b antibodies. There is no proven treatment for MFS and treatment options include plasmapheresis and IVIG.

\section{Bibliography}

[1] C. Fisher, "An unusual variant of acute idiopathic polyuneuritis," Vols. 255(2): 57-65, 1956.

[2] B. C. E. H. P. V. D. M. F. G. e. a. JACOBS, "Serum anti-GQ1b IgG antibodies recognize surface epitopes on Campylobacter jejuni from patients with Miller Fisher syndrome.," Vols. vol. 37, no 2, p. 260-264, 1995.

[3] I. V. A. R. Z. K. D. A. e. a. YEPISHIN, "Miller Fisher syndrome: a case report highlighting heterogeneity of clinical features and focused differ ential diagnosis.," Hawai'i Journal of Medicine \& Public Health, pp. vol. 75, no 7, 2016.

[4] M. K. S. F. T. e. a. MORI, "Clinical features and prognosis of Miller Fisher syndrome," Neurology, pp. vol. 56, no 8, p. 1104-1106, 2001.

[5] L. A. R. V. e. M. N. R. SNYDER, " The Fisher variant of Guillain-Barré syndrome (Fisher syndrome)," Vols. vol. 29, no 4, p. 312-324., 2009.

[6] S. K. Gupta, K. K. Jha, M. D. Chalati and L. T. Alashi, "Case Report: Miller Fisher syndrome," BMJ Case Reports, 2016.

[7] Y. L. LO, " Clinical and immunological spectrum of the Miller Fisher syndrome," Muscle \& nerve, pp. vol. 36, no 5, p. 615-627., 2007.

[8] N. YUKI, " Molecular mimicry between gangliosides and lipopolysaccharides of Campylobacter jejuni isolated from patients with GuillainBarré syndrome and Miller Fisher syndrome.," Vols. vol 176, no Supplement_2, p. S150, 1997.

[9] M. K. S. F. T. e. a. MORI, "Intravenous immunoglobulin therapy for Miller Fisher syndrome," vol. 68, no. no 14, p. 1144-1146., 2007. 